\title{
O PROCESSO DE ELABORAÇÃO DE DOMINÓ PARA ORIENTAÇÃO DE SEGURANÇA NOS CANTEIROS DE CONSTRUÇÃO CIVIL
}

\section{THE PROCESS OF DESIGNING A DOMINO SAFETY ORIENTATION AT BUILDING CONSTRUCTION}

\author{
Darling Kescia Araújo Peixoto Braga ${ }^{1}$, Rosianne Henrique de Freitas Silva ${ }^{1}$, \\ Bruno Noronha Rodrigues ${ }^{2}$, Thiago Brasileiro de Vasconcelos $^{3}$, Maria Aridenise \\ Macena Fontenelle ${ }^{4}$, Raimunda Hermelinda Maia Macena ${ }^{5}$ \\ ${ }^{1}$ Discente do curso de Fisioterapia, Universidade Federal do Ceará (UFC), Fortaleza, Ceará, Brasil. \\ ${ }^{2}$ Discente do curso de Bacharelado em Ciência e Tecnologia, Universidade Federal Rural do Semi-Árido \\ (UFERSA), Mossoró, Rio Grande do Norte, Brasil. \\ ${ }^{3}$ Fisioterapeuta, Discente do curso de Mestrado em Farmacologia, Universidade Federal do Ceará (UFC), \\ Fortaleza, Ceará, Brasil. \\ ${ }^{4}$ Engenheira Civil, Doutorado em Engenharia de Produção, Professora Adjunta da Universidade Federal \\ do Semi-Árido (UFERSA), Mossoró, Rio Grande do Norte, Brasil. \\ ${ }^{5}$ Enfermeira, Doutorado em Ciências Médicas, Professora Adjunta da Universidade Federal do Ceará
} (UFC), Fortaleza, Ceará, Brasil.

\section{RESUMO}

O ambiente de trabalho da construção civil é apontado como um dos principais espaços de incremento da morbimortalidade de trabalhadores no Brasil. Este estudo descreve a experiência de elaboração de um dominó para orientação de segurança nos canteiros de construção civil. Trata-se de um relato de experiência da prática docente extensionista que descreve a elaboração de um dominó educativo realizado em setembro de 2012, a partir de workshops sobre segurança no ambiente da construção civil e produção de material de informação, educação e comunicação em saúde. Com base na filosofia freireana, realizou-se encontros com trabalhadores de chão de obra, seguindo um roteiro de trabalho para a construção do dominó que incluiu a seleção de conteúdos, estilo e formato do material. As mensagens do dominó foram delineadas sobre o formato de imagens compreensíveis e atrativas, e os discentes consideraram-nas adequadas e estimulantes. Esse processo de elaboração para orientação de segurança nos canteiros de construção civil mostrou-se eficaz em estimular novas estratégias educativas, contribuindo com a formação de profissionais de saúde e de engenharia aptos a atuarem no contexto em que estão inseridos.

Palavras-chave: Educação em saúde, Fisioterapia, Engenharia humana, Segurança.

\begin{abstract}
The work environment construction is touted as one of the main areas of increased morbidity and mortality of workers in Brazil. This study describes the experience of designing a domino safety orientation at building construction. This is an experience report of teaching practice-extension that describes the development of a domino education held, in September 2012, from workshops on safety in the construction and production of information materials, health education and communication were raised issues related to this theme. Based on the philosophy of Freire, held meetings with workers of floor work, following a script working to build the domino that included the selection of content, style and format of the material. Messages domino been delineated on the image format understandable and attractive, and students considered it appropriate and stimulating. This preparation process for security guidance at building


construction was effective in stimulating new educational strategies, contributing to the training of health professionals and engineering able to act in the context in which they are inserted.

Key-words: Health education, Physical Therapy, Human Engineering, Safety.

\section{INTRODUÇÃO}

A construção civil, setor de reconhecida importância por sua finalidade e abrangência, é contraditoriamente marcado por um processo de trabalho que propicia, na ausência de ações preventivas, a constante convivência com situações de risco, comprovada pelo elevado número de acidentes, inclusive incapacitantes e fatais (MANGAS, 2003).

O ambiente de trabalho da construção civil tem sido apontado como um dos principais espaços de incremento da morbimortalidade de trabalhadores no Brasil. Para alguns autores, o grande contingente de trabalhadores informais neste ramo associado à baixa escolaridade e ao desconhecimento de seus direitos e deveres tem sido responsável por grande parte dos acidentes (SILVEIRA et al., 2005; MANGAS; GOMEZ; THEDIM-COSTA, 2008).

A construção civil apresenta diversos problemas de ordem gerencial, que inclui falhas na comunicação e gerenciamento. Problemas quanto à gestão da segurança no trabalho são muitas vezes considerados comuns e não despertam as atenções da gerência e dos operários como deveriam, pois outros assuntos tornam-se prioritários (MEDEIROS; RODRIGUES, 2001).

O presente trabalho apresenta a experiência da articulação estabelecida entre docentes e discentes dos cursos de Fisioterapia e Bacharelado em Ciências e Tecnologia (BCT) na busca por estratégias de ensino-aprendizagem para a educação do trabalhador da construção civil ao elaborar um dominó educativo. A parceria se deu entre o curso de Fisioterapia da Universidade Federal do Ceará, através da ação extensionista denominada Programa Promoção da Saúde (PROSA) no Projeto Prosa Coaching, e do Bacharelado em Ciências e Tecnologia (BCT) da Universidade Federal Rural do SemiÁrido, através do programa de extensão FICC (Formação Integrada em Construção Civil) no subcomponente Formação Integrada Em Saúde Na Construção Civil (FICC SAUDÁVEL). 
Tendo em vista que a segurança e a saúde do trabalho na área da construção civil baseiam-se em normas regulamentadoras descritas na Portaria 3214/78 do Ministério do Trabalho e Emprego (MTE), a parceria propunha a realização de atividades de formação mútua com vistas a atender as questões socialmente estabelecidas por cada segmento profissional a fim de propiciar ao campo operativo os conhecimentos adquiridos ao longo da formação, bem como desenvolver habilidades na sociabilização destes conhecimentos para trabalhadores da construção civil, de forma a contribuir para sua autonomia, de modo crítico e consciente, advogando por sua saúde e seus direitos.

Garantir condições dignas de vida e possibilitar que indivíduos e coletivos tenham um maior controle sobre os determinantes da saúde são alguns dos objetivos centrais da Promoção à Saúde (WORLD HEALTH ORGANIZATION, 2005; CARVALHO; GASTALDO, 2008). Um conceito importante na Promoção da Saúde é o empoderamento, que procura possibilitar aos indivíduos e coletivos um aprendizado que os torne capazes de viver a vida em suas distintas etapas e de lidar com as limitações impostas por eventuais enfermidades, sugerindo que estas ações devam ser realizadas em distintos settings, entre os quais a escola, o domicílio, o trabalho e os coletivos comunitários (CARVALHO; GASTALDO, 2008).

O uso de diferentes estratégias e ferramentas educativas na promoção da saúde tomando como pilar teórico a prática educativa-dialógica com base na filosofia freireana tem sido descrita como um elemento importante no processo de empowerment, posto que amplia o conhecimento do indivíduo, melhorando suas atitudes e habilidades, facilitando-lhes a autonomia e lhes ofertando a possibilidade de compreender que as suas próprias ações são capazes de influenciar o seu padrão de saúde (MOREIRA; NÓBREGA; SILVA, 2003; OLIVEIRA et al., 2007; TADDEO et al., 2012). Paulo Freire é citado, neste contexto, como um teórico inspirador de parte da literatura sobre empoderamento produzida por teóricos e profissionais da saúde comprometidos com a mudança social e o fortalecimento de práticas cidadãs questionadoras (GASTALDO, 2005).

Assim, este trabalho se propõe a descrever a experiência do processo de elaboração de dominó para orientação de segurança nos canteiros de construção civil realizado, por docentes e discentes de graduação do curso de Fisioterapia da Universidade Federal do Ceará (CE) e do BCT da Universidade Federal Rural do SemiÁrido $(\mathrm{RN})$. 


\section{DESCRIÇÃO DO RELATO DE CASO}

Trata-se de um relato de experiência da prática docente-extensionista de abordagem qualitativa e interpretativa na área do processo de ensino-aprendizagem em saúde e segurança no ambiente da construção civil.

Este estudo apresenta a descrição das fases de elaboração de um dominó educativo. Para execução do produto piloto foram desenvolvidas duas macro-ações, a saber: 1) Caracterização preliminar das necessidades de informação do público-alvo; 2) O processo de construção do dominó.

Para caracterização preliminar das necessidades de informação do público-alvo foi investigada a literatura disponível em bases virtuais. Nesta etapa, foi possível conhecer as características sócio-demográficas e os fatores de risco para acidentes entre os trabalhadores da construção civil.

O processo de construção do dominó ocorreu no mês de setembro de 2012. Foram realizados dois workshops, a saber: segurança no ambiente da construção civil e produção de material de informação, educação e comunicação em saúde (IEC). Ambos os workshops foram realizados com a presença dos docentes e alunos.

Ao final dos workshops, os discentes foram orientados a produzir um produto piloto de um material didático instrucional dirigido ao tema abordado. Inicialmente, foram desenvolvidas ideias sobre o tema, tendo sido optado pela confecção de um dominó. A seguir, a partir dos temas abordados nos workshops, as informações foram devidamente organizadas e sistematizadas, estabelecendo os déficits de informação prioritários entre o público-alvo. A partir dos resultados encontrados e tendo por base a literatura técnico-científica e experiência profissional foi construído o primeiro protótipo. O material confeccionado foi apresentado ao grupo de extensionistas para avaliação preliminar.

A avaliação do material foi realizada por meio de um instrumento semiestruturado (diário de campo) sobre os aspectos relacionados à organização, estilo de escrita das regras de jogo e design gráfico.

Durante elaboração e implantação de todo o processo de criação do dominó, os autores atuaram como participantes e observadores. As técnicas de coleta de dados utilizadas consistiam em uma observação assistemática. Os dados observados e divulgados tiveram o consentimento dos participantes que participaram dos grupos de trabalho. 


\section{DISCUSSÃO}

$\mathrm{Na}$ caracterização preliminar das necessidades de informação do público-alvo observou-se que a população de trabalhadores da construção civil é adulta com idade média de 35 anos, com baixa escolaridade e estão expostos a inúmeros fatores pessoais e ambientais que ampliam seu risco para acidentes no ambiente laboral. Estes achados nos levaram a considerar a possibilidade de produzir um material de IEC somente com imagens tendo em vista que o nível de escolaridade pode limitar o acesso às informações, devido ao possível comprometimento das habilidades de leitura, escrita, compreensão ou mesmo da fala (FONSECA, 2004; KELLY-SANTOS; ROZEMBERG, 2005).

O processo de construção do dominó teve seu marco inicial na capacitação do grupo e nivelamento dos alunos em relação às temáticas. O primeiro workshop foi conduzido pelos alunos do BCT e abordou os aspectos relativos à segurança no ambiente da construção civil, abordando as regulamentações estabelecidas pela Associação Brasileira de Normas Técnicas (ABNT).

Foram abordadas três normas regulamentadoras estabelecidas pelo Ministério do Trabalho e Emprego, com caráter obrigatório. A NR 06 que determina sobre Equipamentos de Proteção Individual (EPI's), que de acordo com a legislação, é todo dispositivo de uso individual, de fabricação nacional ou estrangeira, destinado a proteger a saúde e a integridade física do trabalhador (MEDEIROS; RODRIGUES, 2001). Esses EPI's são importantes para neutralizar possíveis acidentes contra o corpo do trabalhador, evitar lesões ou minimizar a gravidade delas, além de proteger o corpo contra os efeitos de substâncias tóxicas, alérgicas ou agressivas, que causam doenças ocupacionais. A NR 09 que estabelece o Programa de Prevenção de Riscos Ambientais, que busca a preservação da saúde e da integridade dos trabalhadores, através da antecipação, reconhecimento, avaliação e controle dos riscos ambientais (agentes físicos, químicos e biológicos) do ambiente de trabalho; e a NR 18 que estabelece diretrizes administrativas, de planejamento e de organização para implementar medidas de controle e sistemas preventivos de segurança nos processos, nas condições e no meio ambiente de trabalho na indústria da construção, além de determinar a elaboração do Programa de Condições e Meio Ambiente de Trabalho na Indústria da Construção (PCMAT). 
O segundo workshop foi desenvolvido pelas discentes de Fisioterapia e tratou sobre a produção de material de informação, educação e comunicação em saúde (IEC) e constou de discussões acerca do marco teórico e das etapas para a produção de IEC.

Esses workshops e a elaboração do dominó foram estratégias educativas fundamentais para o trabalho na construção civil. A ausência, na maioria das vezes, de um trabalho educativo intensifica os acidentes, uma vez que impossibilita o conhecimento dos operários aos riscos a que estão se expondo e das suas consequências de exposição a curto e longo prazos. Isso não é novidade, uma vez que também é pertinente ao caráter funcional da concepção de um EPI, que ao ser capaz de neutralizar possíveis condições insalubres do ambiente de trabalho, deve, no entanto, não interferir no desenvolvimento das tarefas laborais do operário. Sendo inadequado, poderá causar tantos danos até maiores do que o próprio risco que procura combater. A educação não deve, porém, ser disposta de forma assustadora e compulsória, pois os trabalhadores bem sabem que as rédeas da segurança não evitarão todos os acidentes (MEDEIROS; RODRIGUES, 2001).

O desenvolvimento do dominó se deu tendo como prerrogativa a possibilidade de ser utilizado nos canteiros de obra durante os intervalos de trabalho. Optou-se pelo uso exclusivo de figuras, pois seria mais acessível e facilitaria a comunicação nãoverbal aos indivíduos com pouca familiaridade com a linguagem escrita. Esse método foi baseado na perspectiva freireana, que estimula o processo ensino-aprendizagem horizontal, dialógico e recíproco (FREIRE, 1988).

O dominó recebeu o nome de Canteiro Seguro sendo confeccionado em folha A4 (210x297mm) em formato de configuração "paisagem". Para facilitar a visualização das figuras, as peças foram confeccionadas tendo $9 \mathrm{~cm}$ de comprimento x 3,6 cm de largura. Foi elaborado um cartão com as regras de como jogar o dominó, a saber: tratase de um dominó em que as peças se complementam, um EPI e a situação que ele pode evitar, por exemplo, o capacete protege do tijolo caindo na cabeça do operário. A mensagem era breve com linguagem simples, considerando que frases longas reduzem a velocidade do processo (THIOLENT, 1986).

Nas pedras do dominó foram utilizados 2 tipos de desenhos, a saber: EPI's (capacete, bota, óculos, cinto de segurança, máscara, uniforme e protetor auricular) e situações de risco nas quais os trabalhadores estarão expostos caso não os utilizem (tijolo caindo na cabeça do operário, operário pisando no prego, substância tóxica 
entrando em contato com os olhos do operário, operário em prédio alto sem proteção, poeira entrando em contato com as vias aéreas do operário, operário com queimadura do sol, barulho de furadeira respectivamente) (ver Ilustração de Algumas Pedras em Anexo A). Estudos revelam a importância da ilustração para atrair o leitor, despertar o interesse e auxiliar na compreensão da mensagem (MOREIRA; NÓBREGA; SILVA, 2003; OLIVEIRA et al., 2007).

Durante a avaliação por pares do material de IEC produzido foram apontados como positivos os aspectos relativos à linguagem, layout e ilustrações. Alguns discentes apresentaram dificuldade em entender a figura relativa ao capacete como material de proteção individual. A apresentação da diagramação foi considerada adequada, com ilustrações dispostas de maneira que o trabalhador da construção civil conseguiria entendê-las, cores foram consideradas atraentes, mas sem deixar o material visualmente poluído.

Os alunos relataram sentir uma dificuldade inicial na elaboração em relação à quais elementos de proteção individual e coletiva seriam abordados, contudo destacaram que essa atividade mostrou a importância do trabalho inter-profissional em intercambiar conhecimentos de sua área temática, considerando a interdependência entre as especialidades (CINTRA; SILVA; CIRCÉIA, 2006).

\section{CONSIDERAÇÕES FINAIS}

O processo de elaboração de um material educativo para orientação de segurança nos canteiros de construção civil mostrou-se eficaz em estimular novas estratégias educativas, na qual os acadêmicos atuam de forma integrada e partilhada, sendo um apoio valioso no desenvolvimento de habilidades durante a formação profissional.

Consideramos, também, que este estudo pôde contribuir com a formação de profissionais de saúde e de engenharia aptos a atuarem no contexto em que estes estão inseridos, com vistas ao suporte integral ao trabalhador da construção civil e à ação interdisciplinar, partindo da pressuposição que a participação do indivíduo possibilita a aquisição de conhecimentos, habilidades e atitudes. 


\section{REFERÊNCIAS}

MANGAS, R. M. N. Acidentes fatais e a desproteção social na construção Civil no Rio de Janeiro [dissertação]. Rio de Janeiro: Fundação Oswaldo Cruz/Escola Nacional de Saúde Pública; 2003. Disponível em:

http://www.arca.fiocruz.br/bitstream/icict/5307/2/623.pdf. Acesso em: 10 de novembro de 2012.

MANGAS, R. M. N.; GOMEZ, C. M.; THEDIM-COSTA, S. M. F. Acidentes de trabalho fatais e desproteção social na indústria da construção civil do Rio de Janeiro. Rev. bras. saúde ocup., v. 33, n. 118, p. 48-55, 2008.

SILVEIRA, C. A.; ROBAZZI, M. L. C. C.; WALTER, E. V.; MARZIALE, M. H. P. Acidentes de trabalho na construção civil identificados através de prontuários hospitalares. Rem: Rev. Esc. Minas, v. 51, n. 1, p. 39-44, 2005.

MEDEIROS, J. A. D. M.; RODRIGUES, C. L. P. A existência de riscos na indústria da construção civil e sua relação com o saber operário. Encontro Nacional de Engenharia de Produção, 2001. Disponível em: http://www.segurancaetrabalho.com.br/download/riscos-alyssonn.pdf. Acesso em: 10 de novembro de 2013.

CARVALHO, S. R.; GASTALDO, D. Promoção à saúde e empoderamento: uma reflexão a partir das perspectivas crítico-social pós-estruturalista. Ciênc. saúde coletiva, v. 13, Supl. 2, p. 2029-40, 2008.

WORLD HEALTH ORGANIZATION (WHO). Ottawa Charter for Health Promotion. 2005. Disponível em: http://www.who.int/hpr/docs/ottawa.htm. Acesso em: 12 de Junho de 2013.

TADDEO, P. S.; GOMES, K. W. L.; CAPRARA, A.; GOMES, A. M. A.; OLIVEIRA, G. C.; MOREIRA, T. M. M. Acesso, prática educativa e empoderamento de pacientes com doenças crônicas. Ciênc. saúde coletiva, v. 17, n. 11, p. 2923-30, 2012. 
OLIVEIRA, V. L. B.; LANDIM, F. L. P.; COLlARES, P. M.; MESQUITA, R. B.; SANTOS, Z. M. S. A. Modelo explicativo popular e profissional das mensagens de cartazes utilizados nas campanhas de saúde. Texto Contexto Enferm., v. 16, n. 2, p. 287-93, 2007.

MOREIRA, M. F.; NÓBREGA, M. M. L.; SILVA, M. I. T. Comunicação escrita: contribuição para a elaboração de material educativo em saúde. Rev Bras Enferm., v. 56, n. 2, p. 184-88, 2003.

GASTALDO, D. Executive Summary of the Research Project: Revisiting Personal is Political. 2005. Disponível em: http://www.nursing.utoronto.ca/immigrationguide. Acesso em: 14 de Junho de 2013.

FONSECA, L. M. M.; SCOCHI, C. G. S.; ROCHA, S. M. M.; LEITE, A. M. Cartilha educativa para orientação materna sobre cuidados com o bebê prematuro. Rev Latinoam Enfermagem, v. 12, n. 1, p. 65-75, 2004.

KELLY-SANTOS, A.; ROZEMBERG, B. Comunicação por impressos na saúde do trabalhador: a perspectiva das instâncias públicas. Ciênc. saúde coletiva, v. 10, n. 4, p. 929-38, 2005.

FREIRE, P. Pedagogia do oprimido. Rio de Janeiro: Paz e Terra, 1988.

THIOLENT, M. Metodologia da Pesquisa-Ação. São Paulo: Cortez Editora, 1986.

CINTRA, S. M. P.; SILVA, C. V.; RIBEIRO, C. A. O ensino do brinquedo/brinquedo terapêutico nos cursos de graduação em enfermagem no Estado de São Paulo. Rev Bras Enferm., v. 59, n. 4, p. 497-501, 2006. 
Ensino, Saúde e Ambiente - V6 (3), pp. 175-184, dez. 2013

ANEXO A
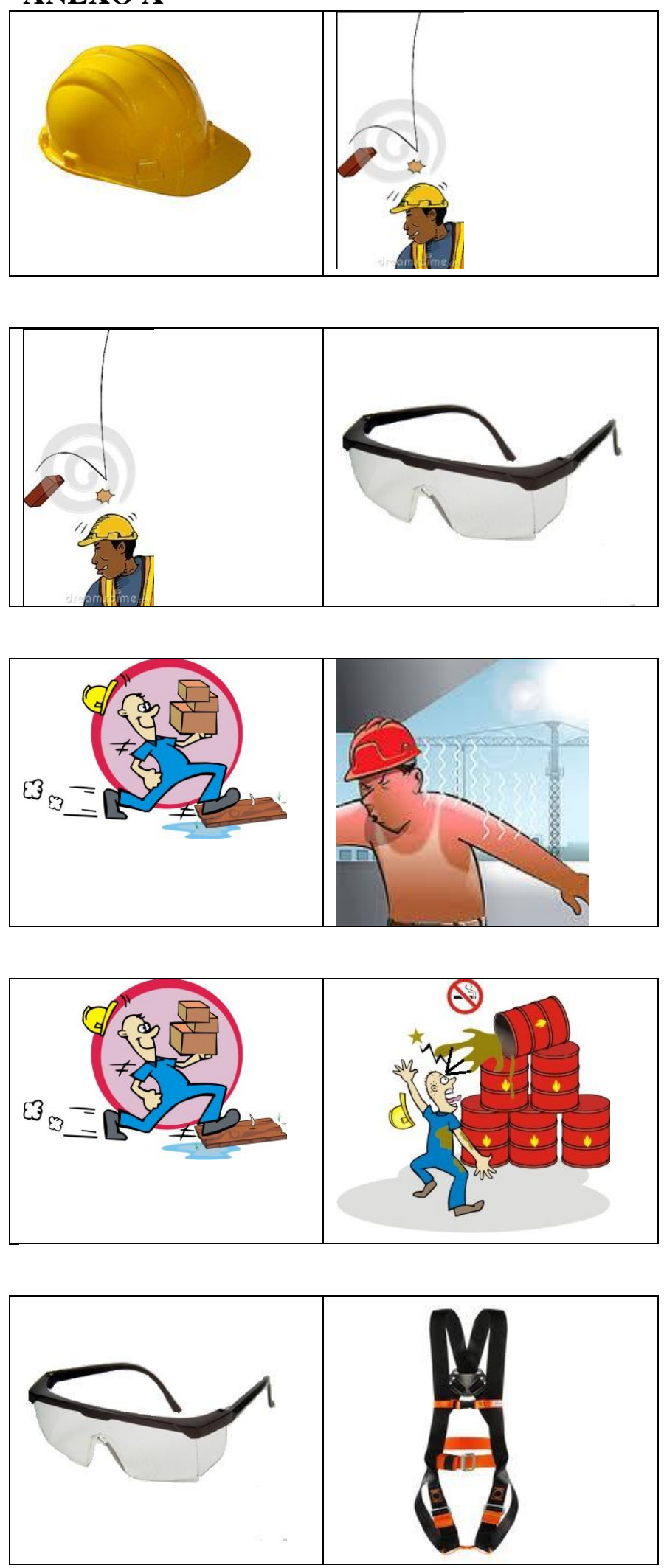